\title{
A Newborn Falsely Suspected of Congenital Hypothyroidism due to Mutated Thyroxine- Binding Globulin with Low Binding Affinity
}

\author{
Rutger C.C. Hengeveld ${ }^{a}$ Monique Albersen ${ }^{b}$ Michael A.H. Hadders ${ }^{c}$ Ilse Hellinga ${ }^{d}$ \\ Hennie Bikker ${ }^{e}$ Annemieke C. Heijboer ${ }^{a}$ b A.S. Paul van Trotsenburg ${ }^{d}$ \\ Jacquelien J. Hillebrand ${ }^{a}$ b Anita Boelen $^{a}$ Nitash Zwaveling-Soonawalad \\ aDepartment of Clinical Chemistry, Amsterdam UMC, Central Diagnostic Laboratory, University of Amsterdam, \\ Amsterdam, The Netherlands; ' Department of Clinical Chemistry, Amsterdam UMC, Endocrine Laboratory, Vrije \\ Universiteit Amsterdam, Amsterdam, The Netherlands; 'Department of Molecular Cancer Research, Oncode Institute, \\ University Medical Center Utrecht, Center for Molecular Medicine, Utrecht, The Netherlands; ${ }^{\mathrm{d} D e p a r t m e n t}$ of Pediatric \\ Endocrinology, Amsterdam UMC, Emma Children's Hospital, University of Amsterdam, Amsterdam, The Netherlands; \\ eDepartment of Clinical Genetics, Amsterdam UMC, University of Amsterdam, Amsterdam, The Netherlands
}

\section{Established Facts}

- Variants in the TBG encoding gene SERPINA7 usually lead to a reduced serum TBG concentration. Hence, TBG deficiency is characterized by reduced TBG, reduced total T4, and normal free T4 concentrations. In rare cases, variants in SERPINA7 cause a functional TBG deficiency leading to decreased T4-binding affinity. In these cases, TBG concentration is normal while total T4 level is reduced.

- The Dutch neonatal screening program for congenital hypothyroidism is T4 based and uses additional TBG measurement to rule out TBG deficiency.

\section{Novel Insights}

- A novel mutation in the SERPINA7 gene resulted in a mutated TBG with normal serum concentration but reduced T4-binding affinity. This led to unnecessary referral of a newborn boy based on an abnormal newborn screening result suggestive of congenital central hypothyroidism (low total T4 and normal TBG).

- While the Dutch screening program includes a TBG measurement to exclude TBG deficiency, it cannot identify such qualitative TBG defects.

\section{Keywords}

Congenital hypothyroidism - Thyroxine-binding globulin deficiency - Thyroxine-binding globulin low affinity .

Neonatal screening

Rutger C.C. Hengeveld, Monique Albersen, and Michael A.H. Hadders contributed equally.

Anita Boelen and Nitash Zwaveling-Soonawala contributed equally.

\section{Abstract}

Introduction: Neonatal screening programs for congenital hypothyroidism $(\mathrm{CH})$ have been implemented worldwide to facilitate early diagnosis and treatment. The Dutch neonatal $\mathrm{CH}$ screening is primarily based on the measurement of thyroxine (T4). When T4 is low, an additional thyroxine-binding globulin (TBG) measurement is performed to reduce the number of false-positive screening results due to harmless karger@karger.com www.karger.com/hrp

Karger $\stackrel{\text { ' }}{5}$ BOPEN ACCESS
(C) 2021 The Author(s)

Published by S. Karger AG, Basel

This is an Open Access article licensed under the Creative Commons Attribution-NonCommercial-4.0 International License (CC BY-NC) (http://www.karger.com/Services/OpenAccessLicense), applicable to the online version of the article only. Usage and distribution for commercial purposes requires written permission.
Correspondence to:

Nitash Zwaveling-Soonawala, n.zwaveling@amsterdamumc.nl 
TBG deficiency. Here, we present a case of a rare functional TBG deficiency leading to a false suspicion of $\mathrm{CH}$. Case Presentation: Neonatal screening in this patient revealed a decreased T4, normal TSH, and normal TBG concentration, suggesting central $\mathrm{CH}$. However, free T4 was normal. DNA sequencing analysis revealed a novel, hemizygous mutation (c.139G >A) in SERPINA7, the gene encoding TBG, resulting in the substitution of the conserved amino acid alanine to threonine at position 27. Crystal structure analyses showed that this substitution has a detrimental effect on binding of $\mathrm{T} 4$ to TBG. Conclusions: The novel SERPINA7 variant in this patient led to a false suspicion of central hypothyroidism in the Dutch T4-based neonatal screening program. It is important to recognize patients with such TBG defects to prevent unnecessary additional testing and treatment.

๑ 2021 The Author(s).
Published by S. Karger AG, Basel

\section{Introduction}

In the circulation, $\mathrm{T} 4$ and $\mathrm{T} 3$ are transported to target tissues bound to the thyroid hormone-binding proteins thyroxine-binding globulin (TBG), albumin, and transthyretin [1]. The main thyroid hormone transporter TBG is encoded by the SERPINA7 gene, located on the $\mathrm{X}$ chromosome. Despite its function in creating a buffer, TBG deficiency is a harmless $\mathrm{X}$-linked trait without clinical hypothyroidism. In affected males, the characteristic blood thyroid hormone profile consists of a low total T4 concentration ( $=$ bound + free fraction of T4) in combination with normal free T4 and TSH concentrations. Complete TBG deficiency is a rare trait with a prevalence of $1: 15,000$ live births while partial TBG defects are more common, 1:4,000 live births [1]. While interpreting thyroid function tests, it is important to recognize innocent TBG deficiency to avoid unnecessary additional diagnostic tests and treatment. This is especially important when interpreting neonatal screening results for congenital hypothyroidism $(\mathrm{CH})$.

Since untreated $\mathrm{CH}$ leads to permanent intellectual and motor disabilities, neonatal screening programs were implemented since the 1970 s to facilitate early detection and treatment [2]. This has proven to be effective in preventing developmental disabilities. Most countries use a TSH-based screening which effectively detects primary $\mathrm{CH}$. However, some countries use a combined total T4 and TSH approach, aiming at detection of both primary and central forms of $\mathrm{CH}$ [3]. A disadvantage of total T4based screening is that it has a rather high rate of false- positive tests due to harmless TBG deficiency. To circumvent this problem, the Dutch newborn screening was adapted into a unique 3 -step screening with an additional TBG measurement [4]. A neonatal screening result with a low total T4, a normal TSH concentration, and accompanied by a low TBG concentration indicates TBG deficiency and requires no further testing. Usually, genetic variants in TBG lead to a reduced plasma TBG concentration and thus a quantitative deficiency. In rare cases, genetic variants cause a functional TBG deficiency: a defective TBG with reduced thyroid hormone-binding affinity (but a normal concentration).

Here, we report a novel variant in SERPINA7 resulting in substitution of a conserved amino acid that has no effect on the protein concentration of TBG but a detrimental effect on the T4-binding affinity. In this case, the mutated TBG led to unnecessary referral for central hypothyroidism in the Dutch neonatal screening program.

\section{Case Presentation}

The male Caucasian newborn patient was born after an uncomplicated pregnancy at 41 1/7 weeks with a birth weight of 4,200 g. Neonatal screening was routinely performed on day 7 , followed by referral to the pediatric endocrinologist because of an abnormal screening result suggestive of congenital central hypothyroidism: decreased total T4 (10 nmol/L blood; $-4.1 \mathrm{SD}$ compared to the daily mean), normal TSH (1 mU/L blood), and a normal TBG (174 $\mathrm{nmol} / \mathrm{L}$ blood) and normal T4/TBG ratio.

According to his parents, the newborn boy was doing well. Upon physical examination, there were no signs or symptoms of hypothyroidism. The family history, including that of a 2 -year-old female sibling, was negative for thyroid or hypothalamic pituitary disease. Thyroid function tests in both parents were normal. Remarkably, in 1992, the mother's brother had an abnormal neonatal screening result for $\mathrm{CH}$, and additional blood investigations had ruled out hypothyroidism. At that time, the uncle's screening result was considered to be false positive.

Additional analyses in venous blood showed normal TSH, FT4, and FT3 levels, excluding $\mathrm{CH}$. Total T4 was low, and total T3 was slightly decreased, while TBG was normal (Table 1) [5]. T3 uptake was increased, indicating that the TBG-binding affinity for T3 is affected in vitro. This finding in combination with normal TBG concentrations was suggestive for impaired binding of T3 and thereby also of T4 to TBG.

Genetic Analysis

Next-generation sequencing analysis revealed a novel hemizygous variant (c.139G>A) in SERPINA7, resulting in substitution of the conserved amino acid alanine to threonine at position 27 (p.(Ala27Thr)) (amino acid numbering based on the mature sequence) (Fig. 1). The variant was rare and not observed in Gno$\mathrm{mAD}$. The validity of the variant was visually inspected using the Integrative Genomics Viewer. Since the TBG concentration was normal, the mutation did not affect TBG protein expression. DNA 


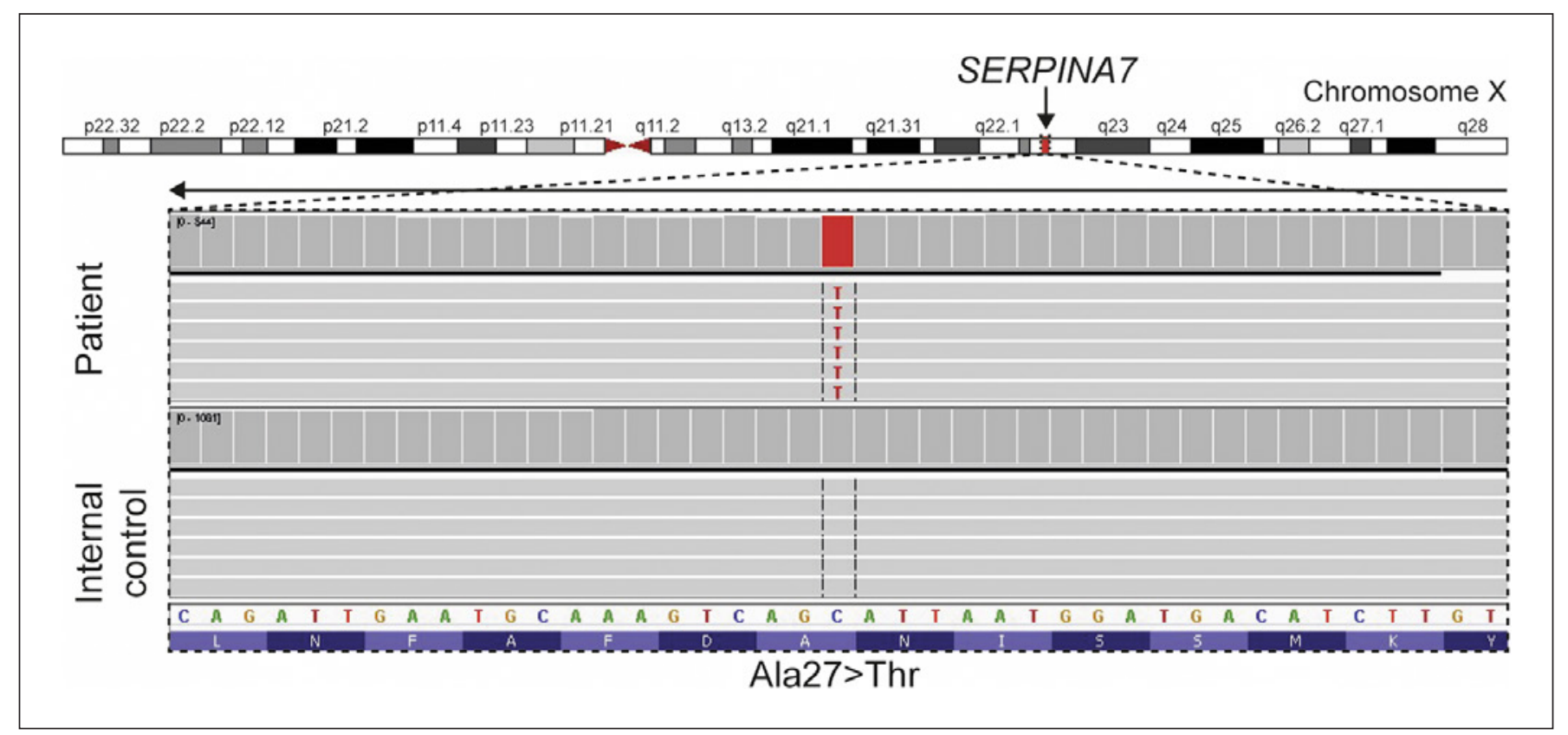

Fig. 1. DNA sequencing analysis revealing a novel, hemizygous mutation (c.139G $>$ A) in SERPINA7, the gene encoding TBG, resulting in the substitution of the amino acid alanine to threonine at position 27 . TBG, thyroxinebinding globulin.

Table 1. Thyroid function test results of the newborn at the first presentation (age 12 days) and at follow-up (age 40 days), both without treatment

\begin{tabular}{|c|c|c|c|}
\hline Parameter & $\begin{array}{l}\text { Age: } \\
12 \text { days }\end{array}$ & $\begin{array}{l}\text { Age: } \\
40 \text { days }\end{array}$ & Reference interval (age) \\
\hline $\mathrm{TSH}, \mathrm{mU} / \mathrm{L}$ & 6.5 & 3.7 & $\begin{array}{l}1.4-8.6 \text { ( } 13-15 \text { days) [5] } \\
0.5-5.0 \text { (>16 days) }\end{array}$ \\
\hline $\mathrm{T} 4, \mathrm{nmol} / \mathrm{L}$ & 25 & 25 & $70-150$ \\
\hline $\mathrm{fT} 4, \mathrm{pmol} / \mathrm{L}$ & 19.7 & 16.1 & $\begin{array}{l}15.3-26.5 \text { ( } 13-15 \text { days) [5] } \\
11.5-28.3 \text { ( } 16 \text { days }-3 \text { months) }\end{array}$ \\
\hline fT4 index (na) & nd & 33 & $60-160$ \\
\hline $\mathrm{T} 3, \mathrm{nmol} / \mathrm{L}$ & 0.95 & 1.25 & $1.3-2.7$ \\
\hline $\mathrm{fT} 3, \mathrm{pmol} / \mathrm{L}$ & nd & 4.0 & 2.3-6.4 (6 days-4 months) \\
\hline $\mathrm{rT} 3, \mathrm{nmol} / \mathrm{L}$ & 0.45 & nd & $0.11-0.44$ \\
\hline T3 uptake (na) & nd & 1.33 & $0.84-1.11$ \\
\hline $\mathrm{Tg}, \mathrm{pmol} / \mathrm{L}$ & 44.4 & nd & $20-525$ \\
\hline TBG, nmol/L & 470 & 460 & $\begin{array}{l}370-750(<14 \text { days old }) \\
200-650(>14 \text { days old })\end{array}$ \\
\hline
\end{tabular}

TSH (Cobas; Roche Diagnostics: ref. 11731459 122), T4 (inhouse RIA), fT4 (Cobas; Roche Diagnostics: ref. 07976836 190), T3 (in-house RIA), fT3 (Architect; Abbott Diagnostics: ref. 7K63), reverse T3 (rT3; in-house RIA), T3 uptake (RIA; Beckman Coulter: ref. A30282), thyroglobulin (Tg) (Kryptor; Thermo Scientific: ref. 832.075), and TBG (RIA, Thermo Scientific: ref. BM00104431). na, not applicable; nd, not determined; TBG, thyroxine-binding globulin. sequences of $A L B$ and TTR were unaffected. Since it did not have any clinical consequences, genetic testing of parents was not performed.

\section{Crystal Structure Analysis}

Structural analysis of human TBG (RCSB Protein Data Bank ID: 2CEO) was performed using the molecular visualization software PyMOL (DeLano, 2002) [6]. All figures were also generated with PyMOL. T3 and T4 bind to a hydrophobic pocket in TBG that is formed by helices $\mathrm{A}$ and $\mathrm{H}$ and by the beta strands $3-5$ (Fig. 2a, b) [6]. Structural analysis revealed that $\mathrm{Ala}^{27}$ is located on helix A at the base of this pocket. The side chain of $\mathrm{Ala}^{27}$ normally points to the inside of the pocket, where it directly interacts with T3/T4 (Fig. 2b). Substitution of $\mathrm{Ala}^{27}$ by Thr results in a steric clash between either $\mathrm{O}^{\gamma 1}$ or $\mathrm{C}^{\gamma 2}$ of $\mathrm{Thr}^{27}$ and $\mathrm{I}^{3 / 4}$ of $\mathrm{T} 3 / 4$, thereby hindering the binding of T3/4 to TBG (Fig. 2c-f).

\section{Discussion}

In this clinical case, a mutated TBG led to unnecessary referral of a newborn boy based on an abnormal neonatal screening result suggestive of congenital central hypothyroidism. While the Dutch screening program includes a TBG measurement to exclude harmless TBG deficiency, it cannot identify functional TBG defects.

The puzzling thyroid function test results in this case consisted of a low total T4 and T3 concentration together 


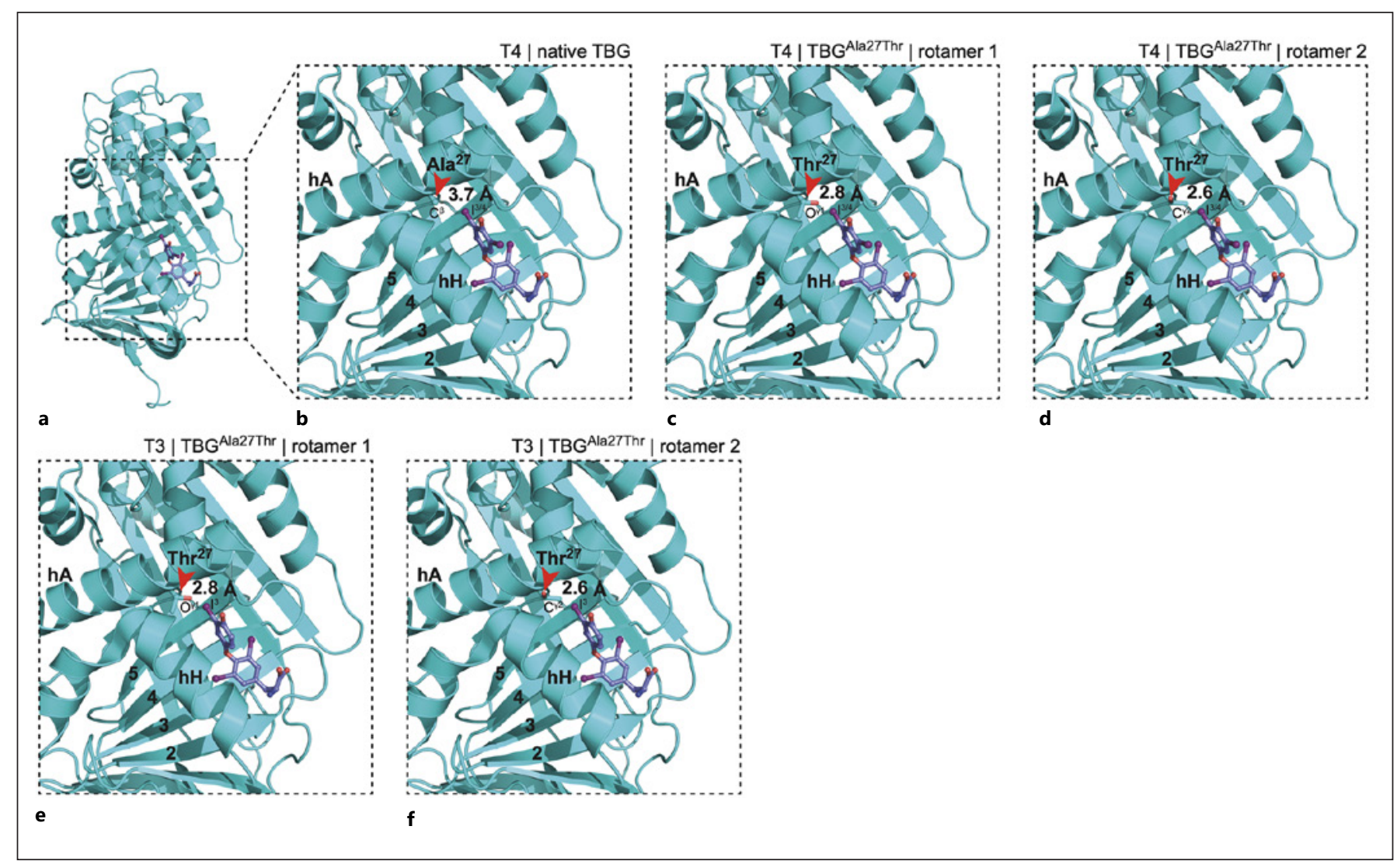

Fig. 2. a, b Protein structure of human native TBG (cyan) bound to T4 (blue). TBG ${ }^{\text {Ala27Thr }}$ rotamer 1 or 2 bound to T4 $(\mathbf{c}, \mathbf{d})$ and rotamer 1 or 2 bound to T3 $(\mathbf{e}, \mathbf{f})$. $\mathbf{c - f}$ Substitution of Ala ${ }^{27}$ by Thr results in a steric clash hindering the binding of T3/4 to TBG. TBG, thyroxine-binding globulin.

with normal TSH, FT4, FT3, and TBG concentrations. Additional analysis including gene sequencing and crystal structure analysis revealed a novel variant in SERPINA7 with no effect on the protein concentration but a detrimental effect on the T3- and T4-binding affinity of TBG. Further functional tests are necessary to better characterize the functional abnormality of the novel SERPINA7 variant. Since TBG deficiency is an X-linked trait, the mother may be a carrier, and in that case, the boy's uncle (mother's brother) may also carry the same SERPINA7 variant and that may have led to his abnormal neonatal $\mathrm{CH}$ screening result in the past. Since we did not test the mother for carriership and since the uncle's medical details could not be retrieved, this remains speculative.

We describe a novel structural variant of TBG; however, such structural variants without significant decrease in serum concentration of TBG are not that uncommon. For example, TBG-A with a reduced affinity for T4 has an allele frequency of 50\% in Australian Aborigines [1]. The amino acid substitution in this case, $\mathrm{Ala}^{27} \mathrm{Thr}$, is located adjacent to $\mathrm{Ser}^{23}$, which is involved in a previously described variant known as TBG-San Diego ( $\mathrm{Ser}^{23} \mathrm{Thr}$ ). TBG-San Diego also affects T3/4 binding by steric hindrance as a result of an extra methyl group in threonine. Males with this variant have low T4 and FT4 index values and have been described as being clinically euthyroid [68].

The Dutch neonatal screening consists of a unique 3-step screening, including additional TBG measurement [4]. The introduction of TBG in the Dutch neonatal screening program since 1995 serves a dual purpose. First, it reduces the number of false positives due to TBG deficiency, and second, calculation of the T4/TBG ratio provides an indirect measure of the FT4 concentration, thereby optimizing the detection of central hypothyroidism. As a result, the Netherlands has one of the world's highest central $\mathrm{CH}$ prevalence of 1 in 16,000 newborns. 
In about $80 \%$ of patients with central $\mathrm{CH}$, multiple pituitary hormone deficiencies are present including potentially life-threatening central adrenal insufficiency [9]. The value of T4-based screening programs in detecting patients with central $\mathrm{CH}$ within the framework of multiple pituitary hormone deficiencies has been recognized and may be implemented in more countries in the future $[3,10]$. It is important to realize that TBG deficiency is an important source of false positives in T4-based screening programs, and as illustrated in this case, even the addition of TBG to the screening algorithm does not completely circumvent this problem since functional TBG deficiencies remain undetected.

In summary, the reported novel variant in SERPINA7 (c.139G >A; p.Ala27Thr) reduces T4- and T3-binding affinity of TBG while TBG concentration is normal. Recognizing such TBG variants is important to avoid unnecessary tests and treatment.

\section{Statement of Ethics}

The investigations in this case report were performed as part of standard health care. Ethical approval for investigating this case was not required in accordance with our institutional guidelines
(Amsterdam UMC, Amsterdam, The Netherlands). Written informed consent was obtained from both parents of the patient for publication of this case report and any accompanying images.

\section{Conflict of Interest Statement}

The authors have no conflicts of interest to declare.

\section{Funding Sources}

The authors did not receive any funding for the work presented in this article.

\section{Author Contributions}

All authors had a role in unraveling the diagnosis in the presented case report. R.C.C.H., M.A., I.H., A.C.H., A.S.P.v.T., J.J.H., A.B., and N.Z.-S. were involved in performing and interpreting biochemical studies; M.A.H.H. and H.B. performed genetic and crystal structure analysis; R.C.C.H., M.A., M.A.H.H., A.B., and N.Z.-S. drafted the first manuscript. All authors discussed previous versions of the manuscript and agreed to the submission of the final version.

\section{References}

1 Mimoto MS, Refetoff S. Clinical recognition and evaluation of patients with inherited serum thyroid hormone-binding protein mutations. J Endocrinol Invest. 2020;43(1):31-41.

2 Ford G, LaFranchi SH. Screening for congenital hypothyroidism: a worldwide view of strategies. Best Pract Res Clin Endocrinol Metab. 2014;28(2):175-87.

3 Leger J, Olivieri A, Donaldson M, Torresani T, Krude H, van Vliet G, et al. European society for paediatric endocrinology consensus guidelines on screening, diagnosis, and management of congenital hypothyroidism. J Clin Endocrinol Metab. 2014;99(2):363-84.

4 Lanting CI, van Tijn DA, Loeber JG, Vulsma T, de Vijlder JJ, Verkerk PH. Clinical effectiveness and cost-effectiveness of the use of the thyroxine/thyroxine-binding globulin ratio to detect congenital hypothyroidism of thyroidal and central origin in a neonatal screening program. Pediatrics. 2005;116(1): $168-73$.

5 Naafs JC, Heinen CA, Zwaveling-Soonawala $\mathrm{N}$, van der Schoor SRD, van Tellingen $\mathrm{V}$, Heijboer AC, et al. Age-specific reference intervals for plasma free thyroxine and thyrotropin in term neonates during the first two weeks of life. Thyroid. 2020;30(8):1106-11.

6 Zhou A, Wei Z, Read RJ, Carrell RW. Structural mechanism for the carriage and release of thyroxine in the blood. Proc Natl Acad Sci U S A. 2006;103(36):13321-6.

7 Sarne DH, Refetoff S, Nelson JC, Linarelli LG. A new inherited abnormality of thyroxine-bind- ing globulin (TBG-San Diego) with decreased affinity for thyroxine and triiodothyronine. J Clin Endocrinol Metab. 1989;68(1):114-9.

8 Bertenshaw R, Sarne D, Tornari J, Weinberg $\mathrm{M}$, Refetoff S. Sequencing of the variant thyroxine-binding globulin (TBG)-San Diego reveals two nucleotide substitutions. Biochim Biophys Acta. 1992;1139(4):307-10.

9 van Tijn DA, de Vijlder JJ, Verbeeten B Jr, Verkerk PH, Vulsma T. Neonatal detection of congenital hypothyroidism of central origin. J Clin Endocrinol Metab. 2005;90(6):3350-9.

10 Zwaveling-Soonawala N, van Trotsenburg AS, Verkerk PH. The severity of congenital hypothyroidism of central origin should not be underestimated. J Clin Endocrinol Metab. 2015;100(2):E297-300. 